\title{
Monitoring the Clinical Trial
}

\author{
Michel Bourin* \\ Neurobiology of mood disorders, University of Nantes, France
}

Received: January 21, 2018; Accepted: February 01, 2018; Published: February 06, 2018

${ }^{*}$ Corresponding author: Michel Bourin, Neurobiology of mood disorders University of Nantes, 98, rue Joseph Blanchart, 44100, Nantes, France, E-mail: michel.bourin@univ-nantes.fr

\begin{abstract}
The monitoring of clinical trials is an essential function for the smooth running of studies. The monitor or the clinical research assistant, in charge of this activity and a real link between the investigator and the sponsor, will have to ensure the respect of the subjects' rights, the reliability and the traceability of the data generated, and to verify that the study is conducted in accordance with the protocol, good clinical practice (GCP) and applicable clinical regulation. Beyond these control objectives, monitoring is also a prevention tool to minimize or avoid potential problems during the study and to anticipate their too late identification, which could lead to more critical consequenceson the conformity of the study. The objective of this paper is to review the various stages of the monitoring and then to give practical tools that can be used at each of these stages.
\end{abstract}

Keywords: Clinical Trial; Clinical Research Assistant; Coordinator; Good Clinical Practice;

\section{Introduction}

A clinical trial is a time-consuming and costly undertaking requiring effective coordination. One of the most important needs when the trial is initiated is to define the monitoring strategy. The quality of the protocol will ensure the scientific value of the trial, but monitoring guarantees the reliability of the data and the performance. Although monitoring may focus on certain special aspects of the trial, four basic points require periodic control: that the trial is proceeding according to the protocol, that the means implemented are adequate, that the investigator and all other participants in the study are fulfilling their various obligations scrupulously, and that the data are relevant and complete.A sophisticated protocol can never compensate for poor quality of the data. Monitoring is thus of major importance, requiring a coordination and management of field organization compatible with good clinical practices [1]. The results can only be validated (or not) once all these obligations have been satisfied [2].

\section{The Coordinator and Clinical Research Assistants}

The coordinator, generally a physician selected and empowered by the promoter, is responsible for ensuring the management and supervision of the trial.

The position of clinical research assistant (CRA) was created in the early 1970s in France. The pharmaceutical industry has made frequent use of CRAs to supervise clinical trials under the direction of the coordinator. As the essential role of the CRA is to ensure the quality and authenticity of the scientific data collected, he must have an excellent knowledge of trial methodology. Accordingly, a program leading to an interuniversity degree was created several years ago to train CRAs.

The CRA should detect any problems that might occur for investigators, provide solutions and especially explain and apply the protocol as well as all the procedures and decisions of the trial coordinator. The CRA is essential to the management of a clinical trial since he can very quickly detect any divergences.

\section{The Monitoring Function}

The coordinator's role begins well before the stage at which the various investigators are involved in the trial. The protocol must be developed in agreement with the different partners. The methodologists, statisticians, pharmacists and investigators must be represented, which is generally ensured by the coordinator. Once the protocol has been elaborated, the investigators should give their opinion before the definitive version is written up in order to be sure that the conditions for carrying out the trial are applicable in all centers and that no overly complicated or unnecessary requirements exist relative to the main objective. If this procedure is followed, it is to be hoped that each of the partners participating in the definition of the objectives of the study and the means to achieve them will be more motivated and respect the protocol better. As the CRAs are more involved in field work and thus closer to the investigators, they should be present during the first meetings in which the trial is worked up.

\section{Training and Informing Investigators}

The choice of investigators, although within the competence of the promoter (as indicated by good clinical practices), is generally made by a local coordinator with a good knowledge of the investigators. The choice can also be made by an organization which may however recruit investigators whose abilities are not always known. The coordinator, accompanied by the CRA, will first inform the investigator of the prerequisites, the protocol and especially the obligation to conform to the procedures to be followed, including those pertaining to severe side effects. The role of the coordinator, and generally that of the CRA, is to provide a detailed explanation of the binders or data sheets used for observations to ensure that the investigator understands everything perfectly well.

The coordinator should also ensure that all members of the team 
participating in the trial with the investigator (i.e. other physicians, nurses and secretaries) are familiar with their respective tasks. If in the context of a hospital trial the investigator (department head) cannot be personally responsible for conducting the trial, someone within his team must be designated as the representative for contacts with the CRA. It is the role of the coordinator and the CRA to obtain the written acceptance of the investigator to abide by good clinical practices and to accommodate the regular visits of the CRA. It is necessary to organize information and training sessions for investigators before the trial begins (cf. the section on selection and meetings with investigators). The essential role of the CRA is now to supervise the investigators for whom he is responsible throughout the trial, beginning with the start-up visit. The first step is administrative since the CRA must determine whether the administrative file is in order. The protocol must be signed and the investigator's curriculum vitae attached. The CRA draws up a schedule with the investigator which may relate to telephone contacts but is essentially concerned with future visits. The CRA provides the investigator with the products necessary for conducting the trial, consisting of the drug packages and the materials necessary for performing biological assays [3]. The CRA must ensure that the communication of information between analytic laboratories and/or the different specialists is performed correctly (e.g. in the event of additional X-ray or cardiologic examinations). In the case of a hospital trial, the CRA must be sure to inform the pharmacist and be certain that he has received the products.

\section{Monitoring the Trial Itself}

The role of the CRA is essential to the conduct of the trial. As indicated above, he must perform regular visits to investigators as scheduled. However, he can make additional visits on his own initiative or at the request of an investigator. The CRA must ensure the application and respect of good clinical practices, particularly strict adhesion to the protocol. The CRA collects the trial data after having consulted the files and checked their authenticity and coherence. Access to patient files may raise ethical problems insofar as the CRA is not a physician. Nonetheless, he is subject to the code of professional secrecy. In the event of a dispute, additional procedures may be necessary. In particular, the coordinator, who is a physician, or another physician designated by the promotor, can check a file when required. Access to files makes it possible to confirm the existence of patients and to verify that they have given their written informed consent. It must also be determined that the randomization order has been respected. Further explanations will be necessary if a physician has failed to do so. An error in randomization could cause an imbalance in one of the treatment groups in the event of an untimely discontinuance of the trial or of non-inclusion of some patients. It is also necessary for the CRA to check that the coordinating center is informed of each patient inclusion, which can be done quite simply by the use of reply cards or e-mail. The CRA must be sure that recruitment is regular to avoid any divergences. As disregard for deadlines can lead to deviations prejudicial to the trial, the CRA should regularly remind investigators of the date for the end of inclusions and encourage them to recruit as rapidly as possible. The CRA should also encourage the slower centers and particularly attempt to know why inclusions are not following the planned pace. It is also necessary to check the relevance of inclusions, which must not only concern those patients who satisfy the inclusion and exclusion criteria. The CRA must not only check the authenticity of the data but also their quality by ensuring that the binders are well kept and that the records are readable and indelible. In the event of a mistake in data recording, the investigator must make the necessary rectifications, which are then checked and approved (signed) by the CRA. The CRA should request any data that have been omitted. He also assists in filling in questionnaires for which he sometimes ensures the codification and data acquisition. The role of the CRA is thus to avoid mistakes, omissions and acts of negligence in the monitoring of patients, particularly in the nature, frequency and regularity of appointments. The CRA does everything possible to limit and identify deviations from the protocol. He encourages investigations concerning patients lost to follow-up and attempts to obtain good observance of the protocol [4].

While respecting confidentiality, the CRA ensures that all critical events have been detected and correctly handled. He keeps the coordinator informed, records the events and verifies them for future validation. Hisinquiries about the opening or not of the treatment code by the investigator, which must of course, be done as a last resort. The CRA must also ensure the conservation of drugs in suitable conditions and the storage of documents. He is concerned with the collection and conveying of certain samples for analysis and assay at a central point. For multicenter trials, the CRA is generally responsible for writing the information sheets which allow investigators to follow the progress of the trial. He keeps up-to-date a file containing all of the documents concerning his role, whether the content of telephone calls, reports of visits to investigators or different types of mail. The CRA must ensure that all participants in the trial other than the investigators, i.e. nurses, biologists and specialist physicians, are competent and that they are informed about the nature of the trial. The CRA must be capable of assessing the quality of the work performed in the center for which he is responsible, whether it is a question of collecting data (missing or mistaken data) or of the delay before data are communicated after each visit (which should be relatively short). He must also ensure the observance of treatment and in particular must count the number of treatment units returned. He should also note the number of visits (and possibly examinations) for which the date was changed or the patient did not show up. He must also ensure that the investigator has made an effort to determine what has happened to drop-outs in order to limit the number of patients lost to follow-up. Finally, the CRA can immediately prevent any deviations by ensuring the regularity of inclusions.

\section{Role of the Clinical Research Assistant at the End of the Trial}

Ultimately, the CRA must perform end-of-trial or centerclosing visits, during which he collects the drugs and other trial materials which have not been used. He sees that the last sheets and binders are reviewed and completed and checks that the 
investigators have filed their documents correctly. A meeting is held at the end of the trial, after the blinding has been lifted, to inform each investigator of the treatment received by his patients during the trial [5].

\section{Monitoring From the Point of View of the Investigators}

Monitoring, long regarded by investigators as a police action, is increasingly considered to be a form of assistance. The clinician has come to realize that his diagnostic and treatment skills are not sufficient to ensure adherence to a rigorous methodology. The CRA should not supervise the investigator but help him to perform his various tasks correctly. In fact, the clinical trial is a paid scientific activity, and the manufacturer, like the drug registration authorities, expects a service of high quality. The CRAs are demanding because they are the watchdogs of the procedure. The investigator should realize that it is not demeaning to rely on the competence and availability of a CRA who necessarily knows the procedure better than himself. The CRA should be regarded as a partner and not as a policeman. An investigator who refuses to allow the CRA to examine his files on the pretext of professional secrecy or, what is worse, on the assumption that the CRA is not scientifically qualified, should withdraw from the trial. In fact, the monitoring of a trial requires coordination and standardization, which are only possible when good relations exist among the different partners. Therefore, the terms and conditions of the trial, which actually constitute the protocol itself, must be based on good clinical practices and determine the respective tasks accepted by all participants. The objectives of monitoring are the good functioning of the study and the reliability of its results, which are absolutely essential to ensure that statistical analysis of results is not based on false data [6, 7].

\section{References}

1. Medical Research Council (MRC). Guidelines for GCP. 1998;147.

2. DeMets DL. Current development in clinical trials: issues old and new. Stat Med. 2012;31(25):2944-2954. doi: 10.1002/ sim.5405

3. Molloy SF, Henley P. Monitoring clinical trials: a practical guide. Trop Med Int Health. 2016;21(12):1602-1611. doi: 10.1111/ tmi.12781

4. Tudur Smith C, Hickey H, Clarke M, Blazeby J, Williamson P. The trials methodological research agenda: results from a priority setting exercise. 2014;15:32.

5. Olsen R, Bihlet AR, Kalakou F, Andersen JR. The impact of clinical trial monitoring approaches on data integrity and cost--a review of current literature. Eur J Clin Pharmacol. 2016;72(4):399-412. doi: 10.1007/s00228-015-2004-y

6. Tudur Smith C, Williamson P, Jones A, Smyth A, Hewer SL, Gamble C. Risk-proportionate clinical trial monitoring: an example approach from a non-commercial trials unit. Trials. 2014;15:127. doi: 10.1186/1745-6215-15-127

7. Baer AR, Devine S, Beardmore CD, Catalano R. Clinical investigator responsibilities. J Oncol Pract. 2011;7(2):124128. doi: 10.1200/JOP.2010.000216 